\title{
Generalized gauge transformations: SU(2) Yang-Mills case
}

\author{
R. Gastmans \\ Institute for Theoretical Physics, University of Leuven, B-3001 Leuven, Belgium \\ Conrad Newton and Tai Tsun Wu \\ Gordon McKay Laboratory, Harvard University, Cambridge, Massachusetts 02138 \\ and Theory Division, CERN, CH-1211 Geneva 23, Switzerland
}

(Received 29 March 1996)

\begin{abstract}
We consider generalized gauge transformations for SU(2) Yang-Mills theory involving products of field operators at different space-time points. The infinitesimal gauge transformations are explicitly constructed to third order in the coupling constant. Also the case of an SU(2) doublet in interaction with the Yang-Mills fields is worked out to third order. [S0556-2821(96)00820-X]

PACS number(s): 11.15.- $\mathrm{q}$
\end{abstract}

\section{INTRODUCTION}

It is the purpose of the present paper to extend our previous work on Abelian gauge theories to the case of the SU(2) Yang-Mills non-Abelian gauge field [1]. This extension is not at all straightforward.

It is well known that, in local quantum field theories, one encounters divergences which arise from taking products of field operators at the same space-time point. As a result, these products do not have a well-defined meaning. More than sixty years ago, Dirac [2] suggested point splitting as a remedy for this difficulty: instead of taking all the field operators at the space-time point $x$, a fixed four-vector $\epsilon$ is introduced so that only field operators with different arguments $(x, x \pm \epsilon, \ldots)$ appear in their product. As long as $\epsilon$ is taken to be different from 0 , the products of field operators is well defined and the theory can be expected to be free of divergences, i.e., the theory is regularized. At the end of the calculation, the limit $\epsilon \rightarrow 0$ is taken, in order to recover the original theory.

A priori, there are many ways in which such a pointsplitting procedure can be implemented [3,4]. For gauge theories, however, one must ensure that the introduction of this new parameter $\epsilon$ does not spoil the invariance under gauge transformations. It thus appears reasonable that one first attempts to construct gauge transformations involving products of field operators taken at different space-time points, which we shall call generalized gauge transformations.

In a previous paper [5], we considered the Abelian case along these lines. To preserve the Abelian character of the generalized transformation, we required that the commutator of two such infinitesimal gauge transformations vanishes. This requirement imposed strong restrictions on the form such a transformation can take. Nevertheless, it was found that such infinitesimal transformations could be constructed and that, for gauge transformations on the fermion fields, they take the form of an infinite series in the coupling constant $e$. This is to be contrasted with the standard U(1) case without point splitting, where the finite gauge transformations on the fermion field are of infinite order in the coupling constant, the infinitesimal ones being only first order in $e$.
In the original paper of Dirac [2], point splitting is used to assign different arguments for the field operators. This convention is followed in our generalized gauge transformation for the Abelian case. The Yang-Mills non-Abelian gauge theory is far more interesting; in particular, such a reassignment of the arguments for the field operators is not enough. Instead, it has been found that it is necessary to average over such arguments. This is discussed in Sec. II.

In this paper, we show how to incorporate the pointsplitting ideas in the gauge transformations for the YangMills case. We basically follow the ideas of the original Yang-Mills paper [1]: we concentrate on the gauge transformations themselves, leaving the derivation of the corresponding Lagrangian for later. At least to third order in the coupling constant $g$, infinitesimal gauge transformations can be constructed which satisfy the condition that the commutator of two such infinitesimal generalized gauge transformations be itself an infinitesimal generalized gauge transformation. To obtain the generalized gauge transformation, we proceed iteratively, i.e., order by order. We see no reason why the iteration procedure could not be worked out to arbitrary order in $g$. Once the pure Yang-Mills case is treated, it becomes relatively straightforward to work out the case of an $\mathrm{SU}(2)$ doublet in interaction with the Yang-Mills fields.

This paper is organized as follows. In Sec. II, we discuss the generalized Yang-Mills gauge transformations and show how the higher order terms in the transformation are to be found. We give the resulting gauge transformation up to order $g^{3}$. In Sec. III, we treat the SU(2) doublet case, and construct the generalized gauge transformations also to order $g^{3}$. Finally, in Sec. IV, we present our conclusions.

\section{PURE YANG-MILLS}

\section{A. Introduction}

Yang-Mills theories are invariant under the infinitesimal gauge transformation

$$
\delta \vec{A}_{\mu}(x)=-\partial_{\mu} \vec{\Lambda}(x)+g \vec{\Lambda}(x) \times \vec{A}_{\mu}(x),
$$

where $\vec{\Lambda}(x)$ is the infinitesimal gauge parameter and $g$ the coupling constant. 
As stated in the Introduction, the product of field operators at the same space-time point does not have a welldefined meaning, and point splitting was introduced by Dirac [2] to remedy this situation. Instead of having $\vec{A}$ and $\vec{\Lambda}$ both at the same point $x$, the arguments of these field operators in a product are taken, for example, to be $x+\epsilon$ and $x-\epsilon$, where $\epsilon$ is a fixed four-vector. Eventually, the limit of $\epsilon \rightarrow 0$ is taken.

Because the field operators are at the same point, the infinitesimal gauge transformation (1) also does not have a well-defined meaning. It is therefore essential to be able to find a generalization of Eq. (1) where point splitting is incorporated. In this section, we show how such a generalized gauge transformation can be obtained in the non-Abelian case.

For that sake, it is convenient to introduce the notation we shall be using. Anticipating the result, we expand the gauge transformation on $\vec{A}_{\mu}(x)$ in powers of $g$, i.e.,

$$
\delta \vec{A}_{\mu}(x)=\sum_{n=0}^{\infty} g^{n} \delta^{(n)} \vec{A}_{\mu}(x) .
$$

Sometimes we use a lower index $\Lambda$ on $\delta_{\Lambda}$ and $\delta_{\Lambda}^{(n)}$ to refer to gauge transformations with a gauge parameters $\vec{\Lambda}$. This is necessary for distinguishing different gauge transformations.

The group property which has to be satisfied then reads

$$
\left[\delta_{\Lambda_{2}}, \delta_{\Lambda_{1}}\right] \vec{A}_{\mu}(x)=\delta_{L} \vec{A}_{\mu}(x)
$$

where $\vec{L}$ is the combined gauge parameter. Also, for this quantity we shall consider a series expansion in the coupling constant:

$$
\vec{L}(x)=\sum_{n=1}^{\infty} g^{n} \vec{L}^{(n)}(x) .
$$

Our goal is to find $\delta^{(n)} \vec{A}_{\mu}(x)$ and $\vec{L}^{(n)}(x)$ which satisfy the group property (3) order by order.

\section{B. Order $g$}

To construct the generalized gauge transformations, we start with the following quite general ansatz:

$$
\begin{gathered}
\delta^{(0)} \vec{A}_{\mu}(x)=-\partial_{\mu} \vec{\Lambda}(x), \\
\delta^{(1)} \vec{A}_{\mu}(x)=\int_{-\infty}^{+\infty} d \xi \rho(\xi) \vec{\Lambda}(x+\xi \epsilon) \times \vec{A}_{\mu}(x-\xi \epsilon) .
\end{gathered}
$$

In Eq. (5), we introduced the weight function $\rho(\xi)$, whose properties will be established by the requirement of the group property (3). To preserve the Hermitian character of the fields $\vec{A}_{\mu}$, we must take $\rho(\xi)$ to be a real function. Also note that, in the limit $\epsilon \rightarrow 0$, our ansatz reduces to the standard Yang-Mills transformations, provided that

$$
\int_{-\infty}^{+\infty} d \xi \rho(\xi)=\text { finite and nonzero. }
$$

Through a redefinition of $g$, we can take $\rho(\xi)$ to be normalized:

$$
\int_{-\infty}^{+\infty} d \xi \rho(\xi)=1
$$

without loss of generality. We shall always use such a normalized $\rho(\xi)$.

To first order in $g$, the group property requires

$$
\left[\delta_{\Lambda_{2}}^{(0)} \delta_{\Lambda_{1}}^{(1)}-\delta_{\Lambda_{1}}^{(0)} \delta_{\Lambda_{2}}^{(1)}\right] \vec{A}_{\mu}(x)=-\partial_{\mu} \vec{L}^{(1)}(x)
$$

The left-hand side (LHS) of this equation reads

$$
\begin{aligned}
-\int_{-\infty}^{+\infty} & d \xi \rho(\xi)\left[\vec{\Lambda}_{1}(x+\xi \epsilon) \times \partial_{\mu} \vec{\Lambda}_{2}(x-\xi \epsilon)\right. \\
& \left.-\vec{\Lambda}_{2}(x+\xi \epsilon) \times \partial_{\mu} \vec{\Lambda}_{1}(x-\xi \epsilon)\right] \\
= & -\partial_{\mu} \int_{-\infty}^{+\infty} d \xi \rho(\xi) \vec{\Lambda}_{1}(x+\xi \epsilon) \times \vec{\Lambda}_{2}(x-\xi \epsilon),
\end{aligned}
$$

provided we take $\rho(\xi)$ to be an even function of $\xi$. In what follows, we shall repeatedly make use of the evenness of $\rho(\xi)$, which we consider to be an important feature of our ansatz (5). By comparison with Eq. (8), we are led to the identification

$$
\vec{L}^{(1)}(x)=\int_{-\infty}^{+\infty} d \xi \rho(\xi) \vec{\Lambda}_{1}(x+\xi \epsilon) \times \vec{\Lambda}_{2}(x-\xi \epsilon)
$$

Already at this order in $g$, one discovers the usefulness of the the weight function $\rho(\xi)$ : it enabled us to find the expression for $\vec{L}^{(1)}$, although the arguments of $\vec{\Lambda}$ and $\vec{A}_{\mu}$ in the gauge transformation (5) are taken at different points. From this analysis, it appears necessary to take $\vec{\Lambda}$ in the first order gauge transformation, Eq. (5), at a space-time point different from $x$, the argument of $\delta^{(1)} \vec{A}_{\mu}(x)$. The essential role of the weight function will become even clearer in the next subsection where we study the gauge transformation to order $g^{2}$.

\section{Order $g^{2}$}

In this order, the formulas become somewhat more complicated. We therefore introduce some more notation in order to limit the size of the formulas and to increase their readability. The group property (3) always refers to commutators of two successive gauge transformations. To avoid writing down commutators, we propose to write only the first term of the commutator, the second term with the $\vec{\Lambda}_{1} \leftrightarrow \vec{\Lambda}_{2}$ interchange being always understood.

Moreover, we shall drop the explicit $x$ dependence in the arguments of $\vec{\Lambda}$ and $\vec{A}_{\mu}$. Thus, $\vec{\Lambda}(\xi \epsilon)$ really stands for $\vec{\Lambda}(x+\xi \epsilon)$, etc.

With these conventions, the expression for $\vec{L}^{(1)}(x)$, e.g., becomes

$$
\vec{L}^{(1)}(0)=\frac{1}{2} \int_{-\infty}^{+\infty} d \xi \rho(\xi) \vec{\Lambda}_{1}(\xi \epsilon) \times \vec{\Lambda}_{2}(-\xi \epsilon) .
$$


Rewritten without these conventions, Eq. (11) would read

$$
\begin{aligned}
\vec{L}^{(1)}(x)= & \frac{1}{2} \int_{-\infty}^{+\infty} d \xi \rho(\xi)\left[\vec{\Lambda}_{1}(x+\xi \epsilon) \times \vec{\Lambda}_{2}(x-\xi \epsilon)\right. \\
& \left.-\vec{\Lambda}_{2}(x+\xi \epsilon) \times \vec{\Lambda}_{1}(x-\xi \epsilon)\right] .
\end{aligned}
$$

To order $g^{2}$, the group property (3) now reads

$$
\begin{aligned}
{\left[\delta_{\Lambda_{2}}^{(1)} \delta_{\Lambda_{1}}^{(1)}+\delta_{\Lambda_{2}}^{(0)} \delta_{\Lambda_{1}}^{(2)}\right] \vec{A}_{\mu}(0)=} & -\partial_{\mu} \vec{L}^{(2)}(0)+\int_{-\infty}^{+\infty} d \xi \rho(\xi) \\
& \times \vec{L}^{(1)}(\xi \epsilon) \times \vec{A}_{\mu}(-\xi \epsilon) .
\end{aligned}
$$

We thus have to find $\vec{L}^{(2)}(0)$ and $\delta^{(2)} \vec{A}_{\mu}(0)$ such that Eq. (13) is satisfied. Let us rewrite Eq. (13) in such a way that the terms which are known from the order $g$ calculation appear on the left-hand side (LHS):

$$
\begin{gathered}
\delta_{\Lambda_{2}}^{(1)} \delta_{\Lambda_{1}}^{(1)} \vec{A}_{\mu}(0)-\int_{-\infty}^{\infty} d \xi \rho(\xi) \vec{L}^{(1)}(\xi \epsilon) \times \vec{A}_{\mu}(-\xi \epsilon) \\
=-\partial_{\mu} \vec{L}^{(2)}(0)-\delta_{\Lambda_{2}}^{(0)} \delta_{\Lambda_{1}}^{(2)} \vec{A}_{\mu}(0) .
\end{gathered}
$$

In Eq. (14), the LHS is explicitly given by

$$
\begin{aligned}
\int & d \xi \int d \eta \rho(\xi) \rho(\eta)\left\{\vec{\Lambda}_{1}(\xi \epsilon) \times\left[\vec{\Lambda}_{2}(-\xi \epsilon+\eta \epsilon) \times \vec{A}_{\mu}(-\xi \epsilon-\eta \epsilon)\right]-\frac{1}{2}\left[\vec{\Lambda}_{1}(\xi \epsilon+\eta \epsilon) \times \vec{\Lambda}_{2}(\xi \epsilon-\eta \epsilon)\right] \times \vec{A}_{\mu}(-\xi \epsilon)\right\} \\
= & \int d \xi \int d \eta \rho(\xi) \rho(\eta)\left\{-\vec{\Lambda}_{1}(\xi \epsilon+\eta \epsilon)\left[\vec{\Lambda}_{2}(-\xi \epsilon) \cdot \vec{A}_{\mu}(\xi \epsilon-\eta \epsilon)\right]-\vec{A}_{\mu}(\xi \epsilon+\eta \epsilon)\left[\vec{\Lambda}_{1}(-\xi \epsilon) \cdot \vec{\Lambda}_{2}(\xi \epsilon-\eta \epsilon)\right]\right. \\
& \left.+\vec{\Lambda}_{1}(\xi \epsilon+\eta \epsilon)\left[\vec{A}_{\mu}(-\xi \epsilon) \cdot \vec{\Lambda}_{2}(\xi \epsilon-\eta \epsilon)\right]\right\},
\end{aligned}
$$

where the integration ranges extend from $-\infty$ to $+\infty$ as usual. Note that in rewriting the expression (15), we again made use of the evenness of the weight function $\rho$.

We first want to find a suitable $\vec{L}^{(2)}$. To this end, we take a zeroth order gauge variation of expression (15) with respect to a third gauge parameter $\vec{\Lambda}_{3}$. Adding together the three terms obtained by cyclically permuting the indices 1,2 , and 3, we find that the integrand of the expression becomes a total derivative

$$
\partial_{\mu}\left\{\vec{\Lambda}_{1}(\xi \epsilon+\eta \epsilon)\left[\vec{\Lambda}_{2}(-\xi \epsilon) \cdot \vec{\Lambda}_{3}(\xi \epsilon-\eta \epsilon)\right]\right\},
$$

where antisymmetrization with respect to the three gauge parameters $\vec{\Lambda}_{i}$ is understood. This suggests that we should take $\vec{L}^{(2)}$ to satisfy

$$
\begin{aligned}
\delta^{(0)} \vec{L}^{(2)}(0)= & -\int d \xi \int d \eta \rho(\xi) \rho(\eta) \vec{\Lambda}_{1}(\xi \epsilon+\eta \epsilon) \\
& \times\left[\vec{\Lambda}_{2}(-\xi \epsilon) \cdot \vec{\Lambda}_{3}(\xi \epsilon-\eta \epsilon)\right] .
\end{aligned}
$$

Equation (17) has a number of solutions, e.g.,

$$
\begin{aligned}
\vec{L}^{(2)}(0)= & \int d \xi \int d \eta \rho(\xi) \rho(\eta) \vec{\Lambda}_{1}(\xi \epsilon+\eta \epsilon) \\
& \times\left[\vec{\Lambda}_{2}(-\xi \epsilon) \cdot \int_{-\xi \epsilon}^{(\xi-\eta) \epsilon} d \tau^{\alpha} \vec{A}_{\alpha}(\tau)\right] .
\end{aligned}
$$

Similar lack of uniqueness has already been seen in the much simpler Abelian case [5]. Here, we choose to take the solution (18) and proceed with the calculation. In the future, when the theory of generalized gauge transformations is further developed, other choices may turn out to be more appropriate. Note that the quantity $\vec{L}^{(2)}(0)$, unlike the $\vec{L}^{(1)}(0)$ of Eq. (11), depends not only on $\vec{\Lambda}_{1}$ and $\vec{\Lambda}_{2}$, but also on $\vec{A}_{\mu}$. For dimensional reasons, this is unavoidable.

In Eq. (18) we introduced the line integral over $\vec{A}$, which has the important property that

$$
\delta^{(0)} \int_{b}^{a} d \tau^{\alpha} \vec{A}_{\alpha}(\tau)=-\vec{\Lambda}(a)+\vec{\Lambda}(b) .
$$

Throughout the entire paper, we shall take all line integrals along straight-line paths going through the point $x$ in the direction of $\epsilon$.

In what follows, we shall use the simplified notation and write

$$
\int_{b}^{a} d \vec{A} \text { for } \int_{x+b}^{x+a} d \tau^{\alpha} \vec{A}_{\alpha}(\tau)
$$

Also note that, following our conventions, we have suppressed the $x$ dependence in the integration limits of the line integral in Eq. (18).

Now that we have obtained $\vec{L}^{(2)}(0)$, we can proceed with finding $\delta^{(2)} \vec{A}_{\mu}(0)$, which is the only remaining unknown quantity in Eq. (14). Using the result (18), we have 


$$
\begin{aligned}
\partial_{\mu} \vec{L}^{(2)}(0)= & \int d \xi \int d \eta \rho(\xi) \rho(\eta) \\
& \times\left\{\partial_{\mu} \vec{\Lambda}_{1}(\xi \epsilon+\eta \epsilon)\left[\vec{\Lambda}_{2}(-\xi \epsilon) \cdot \int_{-\xi \epsilon}^{(\xi-\eta) \epsilon} d \vec{A}\right]\right. \\
& +\vec{\Lambda}_{1}(\xi \epsilon+\eta \epsilon)\left[\partial_{\mu} \vec{\Lambda}_{2}(-\xi \epsilon) \cdot \int_{-\xi \epsilon}^{(\xi-\eta) \epsilon} d \vec{A}\right] \\
& +\vec{\Lambda}_{1}(\xi \epsilon+\eta \epsilon)\left[\vec{\Lambda}_{2}(-\xi \epsilon) \cdot \vec{A}_{\mu}(\xi \epsilon-\eta \epsilon)\right] \\
& -\vec{\Lambda}_{1}(\xi \epsilon+\eta \epsilon)\left[\vec{\Lambda}_{2}(-\xi \epsilon) \cdot \vec{A}_{\mu}(-\xi \epsilon)\right] \\
& \left.+\vec{\Lambda}_{1}(\xi \epsilon+\eta \epsilon)\left[\vec{\Lambda}_{2}(-\xi \epsilon) \cdot \int_{-\xi \epsilon}^{(\xi-\eta) \epsilon} d \vec{F} \mu\right]\right\}
\end{aligned}
$$

where we introduced the shorthand notation

$$
\int_{b}^{a} d \vec{F}_{\mu} \text { for } \int_{x+b}^{x+a} d \tau^{\alpha} \vec{F}_{\mu \alpha}(\tau)
$$

with

$$
\vec{F}_{\mu \nu}=\partial_{\mu} \vec{A}_{\nu}-\partial_{\nu} \vec{A}_{\mu}
$$

The presence of the $\partial_{\mu} \vec{\Lambda}$ terms in Eq. (21) suggests the following terms for $\delta^{(2)} \vec{A}_{\mu}(0)$ :

$$
\begin{gathered}
\int d \xi \int d \eta \rho(\xi) \rho(\eta)\left\{\vec{\Lambda}(\xi \epsilon+\eta \epsilon)\left[\vec{A}_{\mu}(-\xi \epsilon) \cdot \int_{-\xi \epsilon}^{(\xi-\eta) \epsilon} d \vec{A}\right]\right. \\
\left.-\vec{A}_{\mu}(\xi \epsilon+\eta \epsilon)\left[\vec{\Lambda}(-\xi \epsilon) \cdot \int_{-\xi \epsilon}^{(\xi-\eta) \epsilon} d \vec{A}\right]\right\}
\end{gathered}
$$

Indeed, letting $\delta^{(0)}$ act on this expression yields, among other things, the same $\partial_{\mu} \vec{\Lambda}$ terms which are canceled in Eq. (14). Some simple algebra using Eq. (19) shows that only one term remains to be canceled by a second order gauge variation on $\vec{A}_{\mu}$ : namely,

$$
\begin{gathered}
\int d \xi \int d \eta \rho(\xi) \rho(\eta) \vec{\Lambda}_{1}(\xi \epsilon+\eta \epsilon) \\
\times\left[\vec{\Lambda}_{2}(-\xi \epsilon) \cdot \int_{-\xi \epsilon}^{(\xi-\eta) \epsilon} d \vec{F}_{\mu}\right]
\end{gathered}
$$

Consider the following additional contribution to $\delta^{(2)} \vec{A}_{\mu}(0)$ :

$$
\begin{gathered}
\int d \xi \int d \eta \rho(\xi) \rho(\eta)\left\{\vec{\Lambda}(0)\left[\int_{0}^{-\xi \epsilon} d \vec{A} \cdot \int_{-\xi \epsilon}^{(\xi-\eta) \epsilon} d \vec{F}_{\mu}\right]\right. \\
\left.-\left[\vec{\Lambda}(-\xi \epsilon) \cdot \int_{-\xi \epsilon}^{(\xi-\eta) \epsilon} d \vec{F}_{\mu}\right] \int_{0}^{(\xi+\eta) \epsilon} d \vec{A}\right\}
\end{gathered}
$$

Its zeroth order gauge variation cancels the remaining term of Eq. (25), but introduces a new term

$$
\int d \xi \int d \eta \rho(\xi) \rho(\eta) \vec{\Lambda}_{1}(0)\left[\vec{\Lambda}_{2}(0) \cdot \int_{-\xi \epsilon}^{(\xi-\eta) \epsilon} d \vec{F}_{\mu}\right]
$$

This term, however, can be made to vanish if we choose the weight function $\rho$ to satisfy the convolution property

$$
\int_{-\infty}^{+\infty} d \eta \rho(\xi-\eta) \rho(\eta)=\rho(\xi) .
$$

This can be seen as follows. Let $f$ be an arbitrary function. Then,

$$
\begin{aligned}
& \int d \xi \int d \eta \rho(\xi) \rho(\eta)[f(\xi-\eta)-f(-\xi)] \\
& =\int d \xi \int d \eta \rho(\xi+\eta) \rho(\eta) f(\xi)-\int d \xi \rho(\xi) f(-\xi),
\end{aligned}
$$

where for the first term, we shifted the integration variable $\xi$ to $\xi+\eta$, while in the second term, we carried out the $\eta$ integral using the normalization condition (8). For the first term, the $\eta$ integral can now be performed by changing $\eta \rightarrow-\eta$, using the evenness of $\rho(\eta)$ and the convolution property (28). Finally, the evenness of $\rho(\xi)$ has to be invoked to show that the two terms in Eq. (29) cancel. No special property of the arbitrary function $f$ was used in this argument.

Because the remaining term (27) is precisely of the form of the LHS of Eq. (29), we have thus shown that it vanishes.

To conclude this analysis to order $g^{2}$, we summarize our results. We have shown that the second order gauge transformation, the sum of Eqs. (24) and (26),

$$
\begin{aligned}
\delta^{(2)} \vec{A}_{\mu}(0)= & \int d \xi \int d \eta \rho(\xi) \rho(\eta) \\
& \times\left\{\vec{\Lambda}(\xi \epsilon+\eta \epsilon)\left[\vec{A}_{\mu}(-\xi \epsilon) \cdot \int_{-\xi \epsilon}^{(\xi-\eta) \epsilon} d \vec{A}\right]\right. \\
& -\vec{A}_{\mu}(\xi \epsilon+\eta \epsilon)\left[\vec{\Lambda}(-\xi \epsilon) \cdot \int_{-\xi \epsilon}^{(\xi-\eta) \epsilon} d \vec{A}\right] \\
& +\vec{\Lambda}(0)\left[\int_{0}^{-\xi \epsilon} d \vec{A} \cdot \int_{-\xi \epsilon}^{(\xi-\eta) \epsilon} d \vec{F}_{\mu}\right] \\
& \left.-\left[\vec{\Lambda}(-\xi \epsilon) \cdot \int_{-\xi \epsilon}^{(\xi-\eta) \epsilon} d \vec{F}_{\mu}\right] \int_{0}^{(\xi+\eta) \epsilon} d \vec{A}\right\},
\end{aligned}
$$

together with the expression (18) for $\vec{L}^{(2)}(0)$, satisfies the group property of Eq. (13) to order $g^{2}$.

It should be noted that the choice of the zero arguments for the functions $\Lambda_{1}$ and $\Lambda_{2}$ in Eq. (27) is arbitrary. The expression (27) also vanishes if we take arguments for these functions which are independent of $\xi$ and $\eta$. However, this does not seem to lead to essentially new features in the gauge transformations and complicates the writing of the formulas somewhat. We therefore did not pursue this generalization. 
Of course, the formula (30) for the second order gauge transformation would have to be modified accordingly if nonzero arguments are chosen in Eq. (27).

To ensure that the gauge transformations only involve line integrals over finite domains, we had to choose a weight function $\rho$ which is even, normalized in the sense of Eq. (8), and which satisfies the convolution property of Eq. (28). That such a function indeed exists can be seen by considering its Fourier transform:

$$
R(k)=\int_{-\infty}^{+\infty} d \xi e^{i k \xi} \rho(\xi),
$$

where $R(k)$ is an even and real function of $k$ because $\rho(\xi)$ is also an even and real function of $\xi$. In terms of the Fourier transform $R(k)$, the convolution property (28) reads

$$
R(k)^{2}=R(k)
$$

which implies that $R(k)$ is either 0 or 1 . Many such functions exist, e.g.,

$$
\rho(\xi)=\frac{1}{\pi} \frac{\sin \xi}{\xi} .
$$

\section{Order $g^{3}$}

To order $g^{3}$, the expression to be canceled by $\partial_{\mu} \vec{L}^{(3)}$ and $\delta^{(0)} \delta^{(3)} \vec{A}_{\mu}$ reads

$$
\begin{aligned}
\int & d \xi \rho(\xi)\left[\vec{\Lambda}_{1}(\xi \epsilon) \times \delta_{\Lambda_{2}}^{(2)} \vec{A}_{\mu}(-\xi \epsilon)-\vec{L}^{(2)}(\xi \epsilon) \times \vec{A}_{\mu}(-\xi \epsilon)\right]+\int d \xi d \eta \rho(\xi) \rho(\eta)\left\{\vec{\Lambda}_{1}(\xi \epsilon+\eta \epsilon)\right. \\
& \times\left[\delta_{\Lambda_{2}}^{(1)} \vec{A}_{\mu}(-\xi \epsilon) \cdot \int_{-\xi \epsilon}^{(\xi-\eta) \epsilon} d \vec{A}\right]+\vec{\Lambda}_{1}(\xi \epsilon+\eta \epsilon)\left[\vec{A}_{\mu}(-\xi \epsilon) \cdot \int_{-\xi \epsilon}^{(\xi-\eta) \epsilon} \delta_{\Lambda_{2}}^{(1)} \vec{A}\right]-\left[\vec{\Lambda}_{1}(-\xi \epsilon) \cdot \int_{-\xi \epsilon}^{(\xi-\eta) \epsilon} \delta_{\Lambda_{2}}^{(1)} \vec{A}\right] \vec{A}_{\mu}(\xi \epsilon+\eta \epsilon) \\
& -\left[\vec{\Lambda}_{1}(-\xi \epsilon) \cdot \int_{-\xi \epsilon}^{(\xi-\eta) \epsilon} d \vec{A}\right] \delta_{\Lambda_{2}}^{(1)} \vec{A}_{\mu}(\xi \epsilon+\eta \epsilon)+\vec{\Lambda}_{1}(0)\left[\int_{0}^{-\xi \epsilon} \delta_{\Lambda_{2}}^{(1)} \vec{A}_{\mu} \cdot \int_{-\xi \epsilon}^{(\xi-\eta) \epsilon} d \vec{F}_{\mu}\right]+\vec{\Lambda}_{1}(0)\left[\int_{0}^{-\xi \epsilon} d \vec{A}_{\mu} \cdot \int_{-\xi \epsilon}^{(\xi-\eta) \epsilon} \delta_{\Lambda_{2}}^{(1)} \vec{F}_{\mu}\right] \\
& -\left[\vec{\Lambda}_{1}(-\xi \epsilon) \cdot \int_{-\xi \epsilon}^{(\xi-\eta) \epsilon} \delta_{\Lambda_{2}}^{(1)} \vec{F}_{\mu}\right] \int_{0}^{(\xi+\eta) \epsilon} d \vec{A}_{\mu}-\left[\vec{\Lambda}_{1}(-\xi \epsilon) \cdot \int_{-\xi \epsilon}^{(\xi-\eta) \epsilon} d \vec{F}_{\mu}\right] \int_{0}^{(\xi+\eta) \epsilon} \delta_{\Lambda_{2}}^{(1)} \vec{A}^{(3)} \\
& -\vec{L}^{(1)}(\xi \epsilon+\eta \epsilon)\left[\vec{A}_{\mu}(-\xi \epsilon) \cdot \int_{-\xi \epsilon}^{(\xi-\eta) \epsilon} d \vec{A}\right]+\vec{A}_{\mu}(\xi \epsilon+\eta \epsilon)\left[\vec{L}^{(1)}(-\xi \epsilon) \cdot \int_{-\xi \epsilon}^{(\xi-\eta) \epsilon} d \vec{A}\right] \\
- & \left.\vec{L}^{(1)}(0)\left[\int_{0}^{-\xi \epsilon} d \vec{A} \cdot \int_{-\xi \epsilon}^{(\xi-\eta) \epsilon} d \vec{F}_{\mu}\right]+\left[\vec{L}^{(1)}(-\xi \epsilon) \cdot \int_{-\xi \epsilon}^{(\xi-\eta) \epsilon} d \vec{F}_{\mu}\right] \int_{0}^{(\xi+\eta) \epsilon} d \vec{A}\right\}
\end{aligned}
$$

where

$$
\begin{gathered}
\int_{b}^{a} \delta^{(1)} \vec{A}=\int d \lambda \rho(\lambda) \int_{b}^{a} d \tau^{\alpha} \vec{\Lambda}(\tau+\lambda \epsilon) \times \vec{A}_{\alpha}(\tau-\lambda \epsilon) \\
\int_{b}^{a} \delta^{(1)} \vec{F}_{\mu}=\int_{b}^{a} d \tau^{\alpha}\left[\partial_{\mu} \delta^{(1)} \vec{A}_{\alpha}(\tau)-\partial_{\alpha} \delta^{(1)} \vec{A}_{\mu}(\tau)\right]
\end{gathered}
$$

The quantities $\vec{L}^{(1)}$ and $\vec{L}^{(2)}$ in Eq. (34) are given in terms of the gauge parameters $\vec{\Lambda}_{1}$ and $\vec{\Lambda}_{2}$ by Eqs. (11) and (18), respectively.

In analogy with the lower order calculations, we first determine $\vec{L}^{(3)}$. To this end, we find it convenient to rewrite

$$
\int_{b}^{a} \delta^{(1)} \vec{F}_{\mu}=-\delta^{(1)} \vec{A}_{\mu}(a)+\delta^{(1)} \vec{A}_{\mu}(b)+\partial_{\mu} \int_{b}^{a} \delta^{(1)} \vec{A}
$$

Then, letting $\delta^{(0)}$ act on the expression (34), and using the lower order relations

$$
\begin{gathered}
\delta_{\Lambda_{2}}^{(0)} \delta_{\Lambda_{1}}^{(1)} \vec{A}_{\mu}=-\partial_{\mu} \vec{L}^{(1)}, \\
\delta_{\Lambda_{2}}^{(0)} \delta_{\Lambda_{1}}^{(2)} \vec{A}_{\mu}=-\partial_{\mu} \vec{L}^{(2)}-\int d \xi \rho(\xi)\left[\vec{\Lambda}_{1}(\xi \epsilon) \times \delta_{\Lambda_{2}}^{(1)} \vec{A}_{\mu}(-\xi \epsilon)-\vec{L}^{(1)}(\xi \epsilon) \times \vec{A}_{\mu}(-\xi \epsilon)\right],
\end{gathered}
$$

we obtain once more a total derivative: 
$\underline{54}$

GENERALIZED GAUGE TRANSFORMATIONS: SU(2) $\ldots$

5307

$$
\begin{array}{r}
-\partial_{\mu}\left\{\int d \xi \rho(\xi) \vec{\Lambda}_{3}(\xi \epsilon) \times \vec{L}^{(2)}(-\xi \epsilon)\right\}-\partial_{\mu} \iint d \xi d \eta \rho(\xi) \rho(\eta)\left\{\vec{\Lambda}_{3}(\xi \epsilon+\eta \epsilon)\left[\vec{L}^{(1)}(-\xi \epsilon) \cdot \int_{-\xi \epsilon}^{(\xi-\eta) \epsilon} d \vec{A}\right]\right. \\
\left.-\vec{\Lambda}_{1}(\xi \epsilon+\eta \epsilon)\left[\vec{\Lambda}_{2}(-\xi \epsilon) \cdot \int_{-\xi \epsilon}^{(\xi-\eta) \epsilon} \delta_{\Lambda_{3}}^{(1)} \vec{A}\right]-\vec{L}^{(1)}(\xi \epsilon+\eta \epsilon)\left[\vec{\Lambda}_{3}(-\xi \epsilon) \cdot \int_{-\xi \epsilon}^{(\xi-\eta) \epsilon} d \vec{A}\right]\right\} .
\end{array}
$$

This result allows us to find $\vec{L}^{(3)}$ in the same way as we did for orders $g$ and $g^{2}$ :

$$
\begin{aligned}
\vec{L}^{(3)}= & \iiint d \xi d \eta d \lambda \rho(\xi) \rho(\eta) \rho(\lambda)\left\{-\left[\vec{\Lambda}_{1}(-\xi \epsilon) \times \vec{\Lambda}_{2}(\xi \epsilon+\eta \epsilon+\lambda \epsilon)\right]\left[\int_{0}^{(\xi-\eta) \epsilon} d \vec{A} \cdot \int_{0}^{(\xi+\eta-\lambda) \epsilon} d \vec{A}\right]\right. \\
& +\frac{1}{2}\left[\Lambda_{1}(-\xi \epsilon) \times \vec{\Lambda}_{2}(\xi \epsilon+\eta \epsilon+\lambda \epsilon)\right]\left[\int_{0}^{(\xi-\eta) \epsilon} d \vec{A} \cdot \int_{0}^{(\xi-\eta) \epsilon} d \vec{A}\right]+\int_{0}^{(\xi+\eta) \epsilon} d \vec{A}\left[\vec{\Lambda}_{1}(-\xi \epsilon) \cdot \int_{-\xi \epsilon}^{(\xi-\eta) \epsilon} \delta_{\Lambda_{2}}^{(1)} \vec{A}\right] \\
& -\frac{1}{2} \vec{L}^{(1)}(\xi \epsilon+\eta \epsilon)\left[\int_{0}^{-\xi \xi \epsilon} d \vec{A} \cdot \int_{0}^{-\xi \epsilon} d \vec{A}\right]-\int_{0}^{(\xi+\eta) \epsilon} d \vec{A}\left[\vec{L}^{(1)}(-\xi \epsilon) \cdot \int_{-\xi \epsilon}^{(\xi-\eta) \epsilon} d \vec{A}\right]+\frac{1}{2} \vec{L}^{(1)}(-\xi \epsilon)\left[\int_{0}^{(\xi-\eta) \epsilon} d \vec{A} \cdot \int_{0}^{(\xi+\eta) \epsilon} d \vec{A}\right] \\
& +\frac{1}{2}\left[\vec{\Lambda}_{1}(\xi \epsilon-\eta \epsilon) \times \vec{\Lambda}_{2}(-\xi \epsilon+\lambda \epsilon)\right]\left[\int_{0}^{-(\xi+\lambda) \epsilon} d \vec{A} \cdot \int_{0}^{(\xi+\eta) \epsilon} d \vec{A}\right]-\vec{\Lambda}_{1}(0)\left[\int_{0}^{-\xi \epsilon} d \vec{A} \cdot \int_{-\xi \epsilon}^{(\xi-\eta) \epsilon} \delta_{\Lambda_{2}}^{(1)} \vec{A}\right] \\
& -\left[\int_{0}^{-\xi \epsilon} d \vec{A} \times \vec{\Lambda}_{1}(\xi \epsilon+\eta \epsilon+\lambda \epsilon)\right]\left[\vec{\Lambda}_{2}(0) \cdot \int_{0}^{(\xi+\eta-\lambda) \epsilon} d \vec{A}\right] \\
& \left.+\left[\int_{0}^{(\xi-\eta) \epsilon} d \vec{A} \times \vec{\Lambda}_{1}(-\xi \epsilon+\lambda \epsilon)\right]\left[\vec{\Lambda}_{2}(0) \cdot \int_{0}^{-(\xi+\lambda) \epsilon} d \vec{A}\right]\right\} .
\end{aligned}
$$

The third order gauge transformation $\delta^{(3)} \vec{A}_{\mu}$ can now be obtained. Some lengthy algebra shows that we can take

$$
\begin{aligned}
\delta^{(3)} \vec{A}_{\mu}(0)= & \iiint d \xi d \eta d \lambda \rho(\xi) \rho(\eta) \rho(\lambda)\left\{-\int_{0}^{(\xi+\eta) \epsilon} d \vec{A}\left[\vec{A}_{\mu}(-\xi \epsilon) \cdot \int_{-\xi \epsilon}^{(\xi-\eta) \epsilon} \delta^{(1)} \vec{A}\right]\right. \\
& -\left[\vec{\Lambda}(-\xi \epsilon) \times \vec{A}_{\mu}(\xi \epsilon+\eta \epsilon+\lambda \epsilon)+\vec{\Lambda}(\xi \epsilon+\eta \epsilon+\lambda \epsilon) \times \vec{A}_{\mu}(-\xi \epsilon)\right]\left[\int_{0}^{(\xi-\eta) \epsilon} d \vec{A} \cdot \int_{0}^{(\xi+\eta-\lambda) \epsilon} d \vec{A}\right] \\
& +\frac{1}{2}\left[\vec{\Lambda}(-\xi \epsilon) \times \vec{A}_{\mu}(\xi \epsilon+\eta \epsilon+\lambda \epsilon)+\vec{\Lambda}(\xi \epsilon+\eta \epsilon+\lambda \epsilon) \times \vec{A}_{\mu}(-\xi \epsilon)\right]\left[\int_{0}^{(\xi-\eta) \epsilon} d \vec{A} \cdot \int_{0}^{(\xi-\eta) \epsilon} d \vec{A}\right] \\
& +\left[\vec{\Lambda}(-\xi \epsilon+\lambda \epsilon) \times \int_{-\xi \epsilon}^{(\xi-\eta) \epsilon} d \vec{A}\right]\left[\vec{A}_{\mu}(-\xi \epsilon-\lambda \epsilon) \cdot \int_{0}^{(\xi+\eta) \epsilon} d \vec{A}\right] \\
& -\left[\vec{A}_{\mu}(-\xi \epsilon+\lambda \epsilon) \times \int_{-\xi \epsilon}^{(\xi-\eta) \epsilon} d \vec{A}\right]\left[\vec{\Lambda}(-\xi \epsilon-\lambda \epsilon) \cdot \int_{0}^{(\xi+\eta) \epsilon} d \vec{A}\right] \\
& +\left[\vec{\Lambda}(-\xi \epsilon+\lambda \epsilon) \times \vec{A}_{\mu}(\xi \epsilon-\eta \epsilon)\right]\left[\int_{0}^{(\xi+\eta) \epsilon} d \vec{A} \cdot \int_{0}^{-(\xi+\lambda) \epsilon} d \vec{A}\right]-\frac{1}{2} \delta^{(1)} \vec{A}_{\mu}(\xi \epsilon+\eta \epsilon)\left[\int_{0}^{-\xi \epsilon} d \vec{A} \cdot \int_{0}^{-\xi \epsilon} d \vec{A}\right] \\
& +\delta^{(1)} \vec{A}_{\mu}(-\xi \epsilon)\left[\int_{0}^{-\xi \epsilon} d \vec{A} \cdot \int_{0}^{(\xi-\eta) \epsilon} d \vec{A}\right]-\frac{1}{2} \delta^{(1)} \vec{A}_{\mu}(-\xi \epsilon)\left[\int_{0}^{(\xi+\eta) \epsilon} d \vec{A} \cdot \int_{0}^{(\xi-\eta) \epsilon} d \vec{A}\right] \\
& +\vec{A}_{\mu}(0)\left[\int_{0}^{-\xi \xi} d \vec{A} \cdot \int_{-\xi \epsilon}^{(\xi-\eta) \epsilon} \delta^{(1)} \vec{A}\right]+\left[\vec{\Lambda}(\xi \epsilon+\eta \epsilon+\lambda \epsilon) \times \int_{0}^{-\xi \xi} d \vec{A}\right]\left[\vec{A}_{\mu}(0) \cdot \int_{0}^{(\xi+\eta-\lambda) \epsilon} d \vec{A}\right] \\
& -\left[\vec{A}_{\mu}(\xi \epsilon+\eta \epsilon+\lambda \epsilon) \times \int_{0}^{-\xi \epsilon \epsilon} d \vec{A}\right]\left[\vec{\Lambda}(0) \cdot \int_{0}^{(\xi+\eta-\lambda) \epsilon} d \vec{A}\right] \\
& -\left[\vec{\Lambda}(-\xi \epsilon+\lambda \epsilon) \times \int_{0}^{(\xi-\eta) \epsilon} d \vec{A}\right]\left[\vec{A}(0) \cdot \int_{0}^{-(\xi+\lambda) \epsilon} d \vec{A}\right]+\left[\vec{A}_{\mu}(-\xi \epsilon+\lambda \epsilon) \times \int_{0}^{(\xi-\eta) \epsilon} d \vec{A}\right]\left[\vec{\Lambda}(0) \cdot \int_{0}^{-(\xi+\lambda) \epsilon} d \vec{A}\right] \\
& {\left[\vec{A}^{(\xi)}\right] }
\end{aligned}
$$




$$
\begin{aligned}
& +\int_{0}^{(\xi+\eta) \epsilon} \delta^{(1)} \vec{A}\left[\int_{0}^{-\xi \epsilon} d \vec{A} \cdot \int_{-\xi \epsilon}^{(\xi-\eta) \epsilon} d \vec{F}_{\mu}\right]-\int_{0}^{(\xi+\eta) \epsilon} d \vec{F}_{\mu}\left[\int_{0}^{-\xi \epsilon} d \vec{A} \cdot \int_{-\xi \epsilon}^{(\xi-\eta) \epsilon} \delta^{(1)} \vec{A}\right] \\
& -\left[\vec{\Lambda}(-\xi \epsilon) \times \int_{\xi \epsilon}^{(\xi+\eta+\lambda) \epsilon} d \vec{A}\right]\left[\int_{0}^{(\xi-\eta) \epsilon} d \vec{A} \cdot \int_{(\xi-\eta) \epsilon}^{(\xi+\eta-\lambda) \epsilon} d \vec{F}_{\mu}\right] \\
& -\left[\vec{\Lambda}(\xi \epsilon+\eta \epsilon+\lambda \epsilon) \times \int_{0}^{-\xi \epsilon} d \vec{A}\right]\left[\int_{0}^{(\xi+\eta-\lambda) \epsilon} d \vec{A} \cdot \int_{0}^{(\xi-\eta) \epsilon} d \vec{F}_{\mu}\right] \\
& +\left[\vec{\Lambda}(-\xi \epsilon-\lambda \epsilon) \times \int_{0}^{(\xi+\eta) \epsilon} d \vec{A}\right]\left[\int_{0}^{(-\xi+\lambda) \epsilon} d \vec{A} \cdot \int_{0}^{(\xi-\eta) \epsilon} d \vec{F}_{\mu}\right]-\frac{1}{4} \vec{\Lambda}(0)\left[\int_{0}^{-\xi \epsilon} d \vec{F}_{\mu} \cdot \iint_{0}^{(\xi+\eta) \epsilon} \vec{A} \times \vec{A}\right] \\
& +\frac{1}{4} \vec{\Lambda}(0)\left[\int_{0}^{(\xi-\eta) \epsilon} d \vec{F}_{\mu} \cdot \iint_{0}^{-\xi \epsilon} \vec{A} \times \vec{A}\right]-\frac{1}{4} \iint_{0}^{(\xi+\eta) \epsilon} \vec{A} \times \vec{A}\left[\vec{\Lambda}(0) \cdot \int_{-\xi \epsilon}^{(\xi-\eta) \epsilon} d \vec{F}_{\mu}\right] \\
& +\frac{1}{4} \int_{0}^{(\xi-\eta) \epsilon} d \vec{F}_{\mu}\left[\vec{\Lambda}(0) \cdot \iint_{0}^{(\xi+\eta) \epsilon} \vec{A} \times \vec{A}\right]-\frac{1}{4} \int_{0}^{(\xi-\eta) \epsilon} d \vec{F}_{\mu}\left[\vec{\Lambda}(0) \cdot \iint_{0}^{-\xi \epsilon} \vec{A} \times \vec{A}\right] \\
& -\left[\int_{\xi \epsilon}^{(\xi+\eta+\lambda) \epsilon} d \vec{A} \times \int_{0}^{-\xi \epsilon} d \vec{A}\right]\left[\vec{\Lambda}(0) \cdot \int_{(\xi-\eta) \epsilon}^{(\xi+\eta-\lambda) \epsilon} d \vec{F}_{\mu}\right]+\left[\int_{0}^{(\xi+\eta) \epsilon} d \vec{A} \times \int_{0}^{-(\xi+\lambda) \epsilon} d \vec{A}\right]\left[\vec{\Lambda}(0) \cdot \int_{0}^{(\xi-\eta) \epsilon} d \vec{F}_{\mu}\right] \\
& +\frac{1}{2}\left[\int_{\lambda \epsilon}^{(-\xi+\lambda) \epsilon} d \vec{A} \times \int_{0}^{(\xi-\eta) \epsilon} d \vec{F}_{\mu}\right]\left[\vec{\Lambda}(0) \cdot \int_{0}^{-(\xi+\lambda) \epsilon} d \vec{A}\right]+\frac{1}{2}\left[\int_{0}^{-(\xi+\lambda) \epsilon} d \vec{A} \times \int_{0}^{(\xi-\eta) \epsilon} d \vec{F}_{\mu}\right]\left[\vec{\Lambda}(0) \cdot \int_{0}^{\lambda \epsilon} d \vec{A}\right] \\
& -\frac{1}{2}\left[\vec{\Lambda}(0) \times \int_{\lambda \epsilon}^{(\xi+\eta+\lambda) \epsilon} d \vec{A}\right]\left[\int_{0}^{(\xi+\eta-\lambda) \epsilon} d \vec{A} \cdot \int_{0}^{(\xi-\eta) \epsilon} d \vec{F}_{\mu}\right]-\frac{1}{2}\left[\vec{\Lambda}(0) \times \int_{(-\xi+\lambda) \epsilon}^{(\xi+\eta+\lambda) \epsilon} d \vec{A}\right]\left[\int_{0}^{-\lambda \epsilon} d \vec{A} \cdot \int_{0}^{(\xi-\eta) \epsilon} d \vec{F}_{\mu}\right] \\
& +\frac{1}{2}\left[\vec{\Lambda}(0) \times \int_{\lambda \epsilon}^{(-\xi+\lambda) \epsilon} d \vec{A}\right]\left[\int_{0}^{-(\xi+\lambda) \epsilon} d \vec{A} \cdot \int_{0}^{(\xi-\eta) \epsilon} d \vec{F}_{\mu}\right]-\frac{1}{2}\left[\int_{\lambda \epsilon}^{(\xi+\eta+\lambda) \epsilon} d \vec{A} \times \int_{0}^{-\xi \epsilon} d \vec{F}_{\mu}\right]\left[\vec{\Lambda}(0) \cdot \int_{0}^{(\xi+\eta-\lambda) \epsilon} d \vec{A}\right] \\
& \left.-\frac{1}{2}\left[\int_{0}^{(\xi+\eta+\lambda) \epsilon} d \vec{A} \times \int_{0}^{-\xi \epsilon} d \vec{F} \mu\right]\left[\vec{\Lambda}(0) \cdot \int_{0}^{-\lambda \epsilon} d \vec{A}\right]\right\}
\end{aligned}
$$

In Eq. (40) we introduced double line integrals which are defined by

$$
\begin{aligned}
\iint_{a \epsilon}^{b \epsilon} \vec{A} \times \vec{A}= & \frac{1}{2} \int_{(a+\lambda) \epsilon}^{(b+\lambda) \epsilon} d \tau_{1}^{\alpha} \int_{(a-\lambda) \epsilon}^{(b-\lambda) \epsilon} d \tau_{2}^{\beta}\left[\vec{A}_{\alpha}\left(\tau_{1}\right)\right. \\
& \left.\times \vec{A}_{\beta}\left(\tau_{2}\right)\right]\left[1-\operatorname{sgn}\left(\tau_{1}-\tau_{2}-2 \lambda \epsilon\right)\right],
\end{aligned}
$$

where

$$
\operatorname{sgn}\left(\tau_{1}-\tau_{2}-2 \lambda \epsilon\right)=\left\{\begin{array}{lll}
+1 & \text { if } & \tau_{1}-\lambda \epsilon>\tau_{2}+\lambda \epsilon \\
-1 & \text { if } & \tau_{1}-\lambda \epsilon<\tau_{2}+\lambda \epsilon
\end{array}\right.
$$

They are necessary to cancel the line integrals over $\delta^{(1)} \vec{A}$, which appear in Eq. (34). For a discussion of the properties of these double line integrals, see the Appendix.

Equation (39) for $\vec{L}^{(3)}$ and Eq. (40) for $\delta^{(3)} \vec{A}_{\mu}$ are the third order expressions which are needed to satisfy the group property to order $g^{3}$. In their derivation, no new properties of the weight function $\rho$ have to be introduced. We strongly suspect that this analysis can be carried through to arbitrary orders in $g$, but the algebra becomes quite formidable.

\section{SU(2) DOUBLETS}

\section{A. Introduction}

Standard Yang-Mills theories, in which an SU(2) doublet $\psi(x)$ is coupled to Yang-Mills fields, are invariant under the transformations

$$
\begin{gathered}
\delta \vec{A}_{\mu}(x)=-\partial_{\mu} \vec{\Lambda}(x)+g \vec{\Lambda}(x) \times \vec{A}_{\mu}(x), \\
\delta \psi(x)=-i g \frac{1}{2} \vec{\tau} \cdot \vec{\Lambda}(x) \psi(x), \\
\delta \bar{\psi}(x)=\bar{\psi}(x) \text { ig } \frac{1}{2} \vec{\tau} \cdot \vec{\Lambda}(x),
\end{gathered}
$$

where $\vec{\tau}$ are the three Pauli matrices. The group property for the doublet field reads

$$
\left[\delta_{\Lambda_{2}}, \delta_{\Lambda_{1}}\right] \psi(x)=\delta_{L} \psi(x),
$$


where the combined gauge parameter $L$ is the same as for the the Yang-Mills fields. Of course, $\bar{\psi}(x)$ satisfies the same relation (44).

Our aim is to generalize the gauge transformations $\delta \psi$ and $\delta \bar{\psi}(x)$ in such a way that the gauge parameters $\vec{\Lambda}$ and the fields $\psi($ and $\bar{\psi}$ ) are not at the same point $x$. To achieve this goal, we again expand the gauge transformation on $\psi$ in powers of $g$, i.e.,

$$
\begin{gathered}
\delta \psi(x)=\sum_{n=1}^{\infty} g^{n} \delta^{(n)} \psi(x), \\
\delta \bar{\psi}(x)=(\delta \psi(x))^{\dagger} \gamma^{0} .
\end{gathered}
$$

Our task is then to obtain, order by order, the expressions $\delta^{(n)} \psi(x)$ such that the group property (44) is satisfied. We shall limit ourselves to order $g^{3}$.

\section{B. Order $g$}

The ansatz of order $g$, which we propose in analogy with our Abelian paper [5], reads

$$
\delta^{(1)} \psi(x)=-\frac{i}{2} \int_{-\infty}^{+\infty} d \xi \rho(\xi) \vec{\tau} \cdot \vec{\Lambda}(x+\xi \epsilon) \psi(x+2 \xi \epsilon),
$$

where the weight function $\rho(\xi)$ is taken to be the same as in the pure Yang-Mills case. Note that when $\epsilon \rightarrow 0$, we recover the standard case of Eq. (43), because $\rho(\xi)$ is normalized in the sense of Eq. (8).

This ansatz (46) has moreover the desirable property of leaving the mass term in the action for the doublet $\psi$ invariant to first order in $g$. Indeed,

$$
\begin{aligned}
\delta^{(1)} \int & d x \bar{\psi}(x) \psi(x) \\
= & \frac{i}{2} \int d x \int d \xi \rho(\xi)[\bar{\psi}(x+2 \xi \epsilon) \vec{\tau} \cdot \vec{\Lambda}(x+\xi \epsilon) \psi(x) \\
& -\bar{\psi}(x) \vec{\tau} \cdot \vec{\Lambda}(x+\xi \epsilon) \psi(x+2 \xi \epsilon)] \\
= & 0,
\end{aligned}
$$

because we can shift the integration variable $x \rightarrow x-\xi \epsilon$ in both terms, and use the fact that $\rho(\xi)$ is an even function of $\xi$.

\section{Order $g^{2}$}

From now on, we again suppress the $x$ dependence in the arguments of $\vec{\Lambda}$ and $\psi$, as we did in the pure Yang-Mills case. Also, we resort to our convention that commutators of gauge transformations are always understood in our formulas.

To satisfy the group property (44) to order $g^{2}$, we need an expression for $\delta^{(2)} \psi$ such that

$$
\left[\delta_{\Lambda_{2}}^{(1)} \delta_{\Lambda_{1}}^{(1)}-\delta_{L^{(1)}}^{(1)}\right] \psi(0)=-\delta_{\Lambda_{2}}^{(0)} \delta_{\Lambda_{1}}^{(2)} \psi(0),
$$

where $\vec{L}^{(1)}$ is given by Eq. (11) of the pure Yang-Mills case. The LHS of this equation reads

$$
\begin{aligned}
& -\frac{1}{4} \int d \xi \int d \eta \rho(\xi) \rho(\eta) \vec{\tau} \cdot \vec{\Lambda}_{1}(\xi \epsilon) \vec{\tau} \cdot \vec{\Lambda}_{2}(2 \xi \epsilon+\eta \epsilon) \\
& \quad \times \psi(2 \xi \epsilon+2 \eta \epsilon)+\frac{i}{4} \int d \xi \int d \eta \rho(\xi) \rho(\eta) \\
& \quad \times \vec{\tau} \cdot\left[\vec{\Lambda}_{1}(\xi \epsilon+\eta \epsilon) \vec{\Lambda}_{2}(\xi \epsilon-\eta \epsilon)\right] \psi(2 \xi \epsilon) .
\end{aligned}
$$

Using the well-known relation

$$
\tau^{a} \tau^{b}=\delta^{a b}+i \epsilon^{a b c} \tau^{c},
$$

the expression (49) can be rewritten as

$$
\begin{gathered}
\int d \xi \int d \eta \rho(\xi) \rho(\eta)\left\{-\frac{1}{4}\left[\vec{\Lambda}_{1}(\xi \epsilon) \cdot \vec{\Lambda}_{2}(2 \xi \epsilon+\eta \epsilon)\right] \psi(2 \xi \epsilon \epsilon\right. \\
+2 \eta \epsilon)-\frac{i}{4} \vec{\tau} \cdot\left[\vec{\Lambda}_{1}(\xi \epsilon) \times \vec{\Lambda}_{2}(2 \xi \epsilon+\eta \epsilon)\right] \psi(2 \xi \epsilon+2 \eta \epsilon) \\
\left.+\frac{i}{4} \vec{\tau} \cdot\left[\vec{\Lambda}_{1}(\xi \epsilon+\eta \epsilon) \times \vec{\Lambda}_{2}(\xi \epsilon-\eta \epsilon)\right] \psi(2 \xi \epsilon)\right\}
\end{gathered}
$$

This expression is canceled by $\delta_{\Lambda_{2}}^{(0)} \delta_{\Lambda_{1}}^{(2)} \psi(0)$, if we take

$$
\begin{aligned}
\delta^{(2)} \psi(0)= & \int d \xi \int d \eta \rho(\xi) \rho(\eta)\left[-\frac{1}{8}\{[\vec{\Lambda}(\xi \epsilon)\right. \\
& \left.+\vec{\Lambda}(2 \xi \epsilon+\eta \epsilon)] \cdot \int_{\xi \epsilon}^{(2 \xi+\eta) \epsilon} d \vec{A}\right\} \psi(2 \xi \epsilon+2 \eta \epsilon) \\
& -\frac{i}{8} \vec{\tau} \cdot\{[\vec{\Lambda}(2 \xi \epsilon+\eta \epsilon)+\vec{\Lambda}(\xi \epsilon+\eta \epsilon)] \\
& \left.\times \int_{(\xi+\eta) \epsilon}^{\xi \epsilon \epsilon} d \vec{A}\right\} \psi(2 \xi \epsilon+2 \eta \epsilon)-\frac{i}{8} \vec{\tau} \cdot\{[\vec{\Lambda}(\xi \epsilon \\
& \left.+\eta \epsilon)+\vec{\Lambda}(\xi \epsilon)] \times \int_{(\xi+\eta) \epsilon}^{(2 \xi+\eta) \epsilon} d \vec{A}\right\} \psi(2 \xi \epsilon+2 \eta \epsilon) \\
& -\frac{i}{4} \vec{\tau} \cdot\{[\vec{\Lambda}(\xi \epsilon-\eta \epsilon)+\vec{\Lambda}(\xi \epsilon)] \\
& \left.\left.\times \int_{(\xi+\eta) \epsilon}^{\xi \xi} d \vec{A}\right\} \psi(2 \xi \epsilon)\right] .
\end{aligned}
$$

Indeed, all terms in Eq. (51) get canceled, except for introduction of two new terms

$$
\begin{aligned}
& \int d \xi \int d \eta \rho(\xi) \rho(\eta)\left\{-\frac{i}{4} \vec{\tau} \cdot\left[\vec{\Lambda}_{1}(\xi \epsilon+\eta \epsilon)\right.\right. \\
& \left.\times \vec{\Lambda}_{2}(\xi \epsilon+\eta \epsilon)\right] \psi(2 \xi \epsilon+2 \eta \epsilon) \\
& \left.+\frac{i}{4} \vec{\tau} \cdot\left[\vec{\Lambda}_{1}(\xi \epsilon) \times \vec{\Lambda}_{2}(\xi \epsilon)\right] \psi(2 \xi \epsilon)\right\}
\end{aligned}
$$

However, these terms cancel because of the convolution property of the weight functions $\rho$, in complete analogy with the calculations of Eq. (29). 
We thus find that $\delta^{(2)} \psi$ in Eq. (52) has terms without $\vec{\tau}$ as well as terms with $\vec{\tau}$. Also note that, in the limit $\epsilon \rightarrow 0$, the expression for $\delta^{(2)} \psi$ vanishes. Finally, it should be pointed out that $\delta^{(2)} \psi$ leaves the mass term in the action invariant.

\section{Order $g^{3}$}

In order to satisfy the group property of order $g^{3}$, we have to find a suitable third order gauge transformation $\delta^{(3)} \psi$, such that $\delta_{\Lambda_{2}}^{(0)} \delta_{\Lambda_{1}}^{(3)} \psi(0)$ cancels

$$
\begin{aligned}
& {\left[\delta_{\Lambda_{2}}^{(2)} \delta_{\Lambda_{1}}^{(1)}+\delta_{\Lambda_{2}}^{(1)} \delta_{\Lambda_{1}}^{(2)}\right] \psi(0)+\frac{\mathrm{i}}{2} \int d \xi \rho(\xi) \vec{\tau} \cdot \vec{L}^{(2)}(\xi \epsilon) \psi(2 \xi \epsilon)} \\
& \quad+\int d \xi \int d \eta \rho(\xi) \rho(\eta)\left[\frac{1}{8}\left\{\left[\vec{L}^{(1)}(\xi \epsilon)+\vec{L}^{(1)}(2 \xi \epsilon+\eta \epsilon)\right] \cdot \int_{\xi \epsilon}^{(2 \xi+\eta) \epsilon} d \vec{A}\right\} \psi(2 \xi \epsilon+2 \eta \epsilon)\right. \\
& +\frac{i}{8} \vec{\tau} \cdot\left\{\left[\vec{L}^{(1)}(2 \xi \epsilon+\eta \epsilon)+\vec{L}^{(1)}(\xi \epsilon+\eta \epsilon)\right] \times \int_{(\xi+\eta) \epsilon}^{\xi \epsilon} d \vec{A}\right\} \psi(2 \xi \epsilon+2 \eta \epsilon) \\
& +\frac{i}{8} \vec{\tau} \cdot\left\{\left[\vec{L}^{(1)}(\xi \epsilon+\eta \epsilon)+\vec{L}^{(1)}(\xi \epsilon)\right] \times \int_{(\xi+\eta) \epsilon}^{(2 \xi+\eta) \epsilon} d \vec{A}\right\} \psi(2 \xi \epsilon+2 \eta \epsilon) \\
& \left.+\frac{i}{4} \vec{\tau} \cdot\left\{\left[\vec{L}^{(1)}(\xi \epsilon-\eta \epsilon)+\vec{L}^{(1)}(\xi \epsilon)\right] \times \int_{(\xi+\eta) \epsilon}^{\xi \xi \epsilon} d \vec{A}\right\} \psi(2 \xi \epsilon)\right]
\end{aligned}
$$

where $\vec{L}^{(1)}$ and $\vec{L}^{(2)}$ are the combined gauge parameters of the pure Yang-Mills case of order $g$ and $g^{2}$, given by Eqs. (11) and (18).

The cancellation is achieved if we take

$$
\begin{aligned}
\delta^{(3)} \psi(0)= & \frac{1}{16} \int d \xi \int d \eta \int d \lambda \rho(\xi) \rho(\eta) \rho(\lambda)\left[C_{1} \psi(2 \xi \epsilon+2 \eta \epsilon+2 \lambda \epsilon)+C_{2} \psi(2 \xi \epsilon+2 \eta \epsilon)\right. \\
& \left.+i \vec{\tau} \cdot \vec{D}_{1} \psi(2 \xi \epsilon+2 \eta \epsilon+2 \lambda \epsilon)+i \vec{\tau} \cdot \vec{D}_{2} \psi(2 \xi \epsilon+2 \eta \epsilon)+i \vec{\tau} \cdot \vec{D}_{3} \psi(2 \xi \epsilon)\right],
\end{aligned}
$$

with

$$
\begin{aligned}
C_{1}= & \vec{\Lambda}(2 \xi \epsilon+2 \eta \epsilon+\lambda \epsilon) \cdot\left[\int_{(\xi+\eta) \epsilon}^{(2 \xi+\eta) \epsilon} d \vec{A} \times \int_{(\xi+\eta) \epsilon}^{\xi \epsilon} d \vec{A}\right]-\vec{\Lambda}(\xi \epsilon) \cdot\left[\int_{(2 \xi+\eta+\lambda) \epsilon}^{(2 \xi+\eta) \epsilon} d \vec{A} \times \int_{(2 \xi+\eta+\lambda) \epsilon}^{(2 \xi+2 \eta+\lambda) \epsilon} d \vec{A}\right] \\
& +2\left[\vec{\Lambda}(\xi \epsilon) \times \int_{(\xi+\eta+\lambda) \epsilon}^{(2 \xi+2 \eta+\lambda) \epsilon} d \vec{A}+\vec{\Lambda}(\xi \epsilon+\eta \epsilon+\lambda \epsilon) \times \int_{(\xi+\eta+\lambda) \epsilon}^{\xi \epsilon} d \vec{A}\right] \cdot \int_{(\xi+\eta+\lambda) \epsilon}^{(2 \xi+\eta+\lambda) \epsilon} d \vec{A} \\
& -2\left[\vec{\Lambda}(2 \xi \epsilon+2 \eta \epsilon+\lambda \epsilon) \times \int_{(\xi+\eta+\lambda) \epsilon}^{\xi \epsilon} d \vec{A}+\vec{\Lambda}(\xi \epsilon+\eta \epsilon+\lambda \epsilon) \times \int_{(\xi+\eta+\lambda) \epsilon}^{(2 \xi+2 \eta+\lambda) \epsilon} d \vec{A}\right] \cdot \int_{(\xi+\eta+\lambda) \epsilon}^{(\xi+\eta) \epsilon} d \vec{A} \\
& -2 \vec{\Lambda}(\xi \epsilon+\eta \epsilon+\lambda \epsilon) \cdot\left[\int_{(\xi+\eta+\lambda) \epsilon}^{\xi \epsilon} d \vec{A} \times \int_{(\xi+\eta+\lambda) \epsilon}^{(2 \xi+2 \eta+\lambda) \epsilon} d \vec{A}\right], \\
C_{2}= & -\frac{1}{2}[\vec{\Lambda}(\xi \epsilon)+\vec{\Lambda}(2 \xi \epsilon+\eta \epsilon)] \cdot\left[\int_{\xi \epsilon}^{(2 \xi+\eta) \epsilon} \vec{A} \times \vec{A}\right]-2 \vec{\Lambda}(\xi \epsilon+\eta \epsilon) \cdot\left[\int_{(\xi+\eta) \epsilon}^{\xi \epsilon} d \vec{A} \times \int_{(\xi+\eta) \epsilon}^{(2 \xi+\eta) \epsilon} d \vec{A}\right] \\
& +\vec{\Lambda}(\xi \epsilon) \cdot\left[\int_{(\xi-\lambda) \epsilon}^{(2 \xi+\eta) \epsilon} d \vec{A} \times \int_{(\xi+\lambda) \epsilon}^{(2 \xi+\eta+\lambda) \epsilon} d \vec{A}\right]-\vec{\Lambda}(2 \xi \epsilon+\eta \epsilon) \cdot\left[\int_{(2 \xi+\eta-\lambda) \epsilon}^{\xi \xi \epsilon} d \vec{A} \times \int_{(2 \xi+\eta+\lambda) \epsilon}^{(\xi+\lambda) \epsilon} d \vec{A}\right] \\
& +\vec{\Lambda}(\xi \epsilon-\lambda \epsilon) \cdot\left[\int_{\xi \epsilon}^{(2 \xi+\eta) \epsilon} d \vec{A} \times \int_{(\xi+\lambda) \epsilon}^{\xi} d \vec{A}\right]+\vec{\Lambda}(2 \xi \epsilon+\eta \epsilon-\lambda \epsilon) \cdot\left[\int_{\xi \epsilon}^{(2 \xi+\eta) \epsilon} d \vec{A} \times \int_{(2 \xi+\eta+\lambda) \epsilon}^{(2 \xi+\eta) \epsilon} d \vec{A}\right],
\end{aligned}
$$

and 
$\underline{54}$

GENERALIZED GAUGE TRANSFORMATIONS: SU(2) ...

5311

$$
\begin{aligned}
& \vec{D}_{1}=\vec{\Lambda}(2 \xi \epsilon+2 \eta \epsilon+\lambda \epsilon) \times\left[\int_{(\xi+\eta) \epsilon}^{\xi \epsilon} d \vec{A} \times \int_{(\xi+\eta) \epsilon}^{(2 \xi+\eta) \epsilon} d \vec{A}\right]+\vec{\Lambda}(\xi \epsilon) \times\left[\int_{(2 \xi+\eta+\lambda) \epsilon}^{(2 \xi+2 \eta+\lambda) \epsilon} d \vec{A} \times \int_{(2 \xi+\eta+\lambda) \epsilon}^{(2 \xi+\eta) \epsilon} d \vec{A}\right] \\
& +2 \vec{\Lambda}(\xi \epsilon) \times\left[\int_{(\xi+\eta+\lambda) \epsilon}^{(2 \xi+\eta+\lambda) \epsilon} d \vec{A} \times \int_{(\xi+\eta+\lambda) \epsilon}^{(2 \xi+2 \eta+\lambda) \epsilon} d \vec{A}\right]+2 \vec{\Lambda}(2 \xi \epsilon+2 \eta \epsilon+\lambda \epsilon) \times\left[\int_{(\xi+\eta+\lambda) \epsilon}^{(\xi+\eta) \epsilon} d \vec{A} \times \int_{(\xi+\eta+\lambda) \epsilon}^{\xi \epsilon} d \vec{A}\right] \\
& +2 \vec{\Lambda}(\xi \epsilon+\eta \epsilon+\lambda \epsilon) \times\left[\int_{(\xi+\eta+\lambda) \epsilon}^{\xi \epsilon} d \vec{A} \cdot \int_{(\xi+\eta+\lambda) \epsilon}^{(2 \xi+2 \eta+\lambda) \epsilon} d \vec{A}\right]-\frac{1}{2} \vec{\Lambda}(\xi \epsilon)\left[\int_{(2 \xi+\eta) \epsilon}^{(2 \xi+2 \eta+\lambda) \epsilon} d \vec{A} \cdot \int_{(2 \xi+\eta) \epsilon}^{(2 \xi+2 \eta+\lambda) \epsilon} d \vec{A}\right] \\
& -\frac{1}{2} \vec{\Lambda}(2 \xi \epsilon+2 \eta \epsilon+\lambda \epsilon)\left[\int_{(2 \xi+\eta) \epsilon}^{\xi \epsilon} d \vec{A} \cdot \int_{(2 \xi+\eta) \epsilon}^{\xi \epsilon} d \vec{A}\right]-2\left[\vec{\Lambda}(2 \xi \epsilon+2 \eta \epsilon+\lambda \epsilon) \cdot \int_{(\xi+\eta+\lambda) \epsilon}^{(2 \xi+2 \eta+\lambda) \epsilon} d \vec{A}\right] \int_{(\xi+\eta+\lambda) \epsilon}^{\xi \epsilon} d \vec{A} \\
& -2\left[\vec{\Lambda}(\xi \epsilon) \cdot \int_{(\xi+\eta+\lambda) \epsilon}^{\xi \epsilon} d \vec{A}\right] \int_{(\xi+\eta+\lambda) \epsilon}^{(2 \xi+2 \eta+\lambda) \epsilon} d \vec{A}, \\
& \vec{D}_{2}=-\frac{1}{2}[\vec{\Lambda}(2 \xi \epsilon+\eta \epsilon)+\vec{\Lambda}(\xi \epsilon+\eta \epsilon)] \times\left[\iint_{(\xi+\eta) \epsilon}^{\xi \epsilon} \vec{A} \times \vec{A}\right]-\frac{1}{2}[\vec{\Lambda}(\xi \epsilon+\eta \epsilon)+\vec{\Lambda}(\xi \epsilon)] \times\left[\iint_{(\xi+\eta) \epsilon}^{(2 \xi+\eta) \epsilon} \vec{A} \times \vec{A}\right] \\
& +\int_{(\xi+\eta) \epsilon}^{(2 \xi+\eta) \epsilon} d \vec{A} \times\left\{[\vec{\Lambda}(\xi \epsilon-\lambda \epsilon)+\vec{\Lambda}(\xi \epsilon+\eta \epsilon-\lambda \epsilon)] \times \int_{(\xi+\eta+\lambda) \epsilon}^{(\xi+\lambda) \epsilon} d \vec{A}\right\}+\int_{(\xi+\eta) \epsilon}^{\xi \epsilon} d \vec{A} \times\{[\vec{\Lambda}(2 \xi \epsilon+\eta \epsilon-\lambda \epsilon) \\
& \left.+\vec{\Lambda}(\xi \epsilon+\eta \epsilon-\lambda \epsilon)] \times \int_{(\xi+\eta+\lambda) \epsilon}^{(2 \xi+\eta+\lambda) \epsilon} d \vec{A}\right\}-2 \int_{(\xi+\eta) \epsilon}^{(2 \xi+\eta) \epsilon} d \vec{A} \times\left\{[\vec{\Lambda}(\xi \epsilon-\lambda \epsilon)+\vec{\Lambda}(\xi \epsilon)] \times \int_{\xi \epsilon}^{(\xi+\lambda) \epsilon} d \vec{A}\right\}-2 \int_{(\xi+\eta) \epsilon}^{\xi \epsilon} d \vec{A} \\
& \times\left\{[\vec{\Lambda}(2 \xi \epsilon+\eta \epsilon-\lambda \epsilon)+\vec{\Lambda}(2 \xi \epsilon+\eta \epsilon)] \times \int_{(2 \xi+\eta) \epsilon}^{(2 \xi+\eta+\lambda) \epsilon} d \vec{A}\right\}-2\left[\vec{\Lambda}(\xi \epsilon) \cdot \int_{(2 \xi+\eta) \epsilon}^{(\xi+\eta) \epsilon} d \vec{A}\right] \int_{(\xi+\eta) \epsilon}^{(2 \xi+\eta) \epsilon} d \vec{A}-2[\vec{\Lambda}(2 \xi \epsilon+\eta \epsilon) \\
& \left.\cdot \int_{\xi \epsilon}^{(\xi+\eta) \epsilon} d \vec{A}\right] \int_{(\xi+\eta) \epsilon}^{\xi \epsilon} d \vec{A}-2 \vec{\Lambda}(\xi \epsilon+\eta \epsilon)\left[\int_{(\xi+\eta) \epsilon}^{\xi \epsilon} d \vec{A} \cdot \int_{(\xi+\eta) \epsilon}^{(2 \xi+\eta) \epsilon} d \vec{A}\right]+\vec{\Lambda}(\xi \epsilon+\eta \epsilon)\left[\int_{(2 \xi+\eta) \epsilon}^{\xi \epsilon} d \vec{A} \cdot \int_{(2 \xi+\eta) \epsilon}^{\xi \epsilon} d \vec{A}\right] \\
& -2\left[\vec{\Lambda}(\xi \epsilon+\eta \epsilon) \cdot \int_{(\xi+\eta+\lambda) \epsilon}^{\xi \epsilon \epsilon} d \vec{A}\right] \int_{(\xi+\eta-\lambda) \epsilon}^{(\xi-\lambda) \epsilon} d \vec{A}-2\left[\vec{\Lambda}(\xi \epsilon+\eta \epsilon) \cdot \int_{(\xi+\eta+\lambda) \epsilon}^{(2 \xi+\eta) \epsilon} d \vec{A}\right] \int_{(\xi+\eta-\lambda) \epsilon}^{(2 \xi+\eta-\lambda) \epsilon} d \vec{A} \\
& +2\left[\vec{\Lambda}(\xi \epsilon+\eta \epsilon) \cdot \int_{\xi \epsilon}^{(\xi-\lambda) \epsilon} d \vec{A}\right] \int_{(\xi+\eta+\lambda) \epsilon}^{\xi \epsilon} d \vec{A}+2\left[\vec{\Lambda}(\xi \epsilon+\eta \epsilon) \cdot \int_{(2 \xi+\eta) \epsilon}^{(2 \xi+\eta-\lambda) \epsilon} d \vec{A}\right] \int_{(\xi+\eta+\lambda) \epsilon}^{(2 \xi+\eta) \epsilon} d \vec{A} \\
& +4\left[\vec{\Lambda}(\xi \epsilon) \cdot \int_{(\xi+\eta) \epsilon}^{(2 \xi+\eta) \epsilon} d \vec{A}\right] \int_{(\xi+\eta+\lambda) \epsilon}^{\xi \epsilon} d \vec{A}+4\left[\vec{\Lambda}(2 \xi \epsilon+\eta \epsilon) \cdot \int_{(\xi+\eta) \epsilon}^{\xi \epsilon} d \vec{A}\right] \int_{(\xi+\eta+\lambda) \epsilon}^{(2 \xi+\eta) \epsilon} d \vec{A} \\
& -2 \vec{\Lambda}(\xi \epsilon+\eta \epsilon+\lambda \epsilon)\left\{\left[\int_{(\xi+\eta) \epsilon}^{\xi \epsilon} d \vec{A}\right]^{2}+\left[\int_{(\xi+\eta) \epsilon}^{(2 \xi+\eta) \epsilon} d \vec{A}\right]^{2}-\left[\int_{\xi \epsilon}^{(2 \xi+\eta) \epsilon} d \vec{A}\right]^{2}\right\}
\end{aligned}
$$

$$
\begin{aligned}
\vec{D}_{3}= & -[\vec{\Lambda}(\xi \epsilon-\eta \epsilon)+\vec{\Lambda}(\xi \epsilon)] \times\left[\iint_{(\xi+\eta) \epsilon}^{\xi \epsilon} \vec{A} \times \vec{A}\right]+2\left[\vec{\Lambda}(\xi \epsilon-\eta \epsilon+\lambda \epsilon) \times \int_{(\xi-\lambda) \epsilon}^{(\xi-\eta-\lambda) \epsilon} d \vec{A}\right] \times \int_{(\xi+\eta) \epsilon}^{\xi \epsilon} d \vec{A} \\
& +2\left[\vec{\Lambda}(\xi \epsilon+\lambda \epsilon) \times \int_{(\xi+\eta-\lambda) \epsilon}^{(\xi-\lambda) \epsilon} d \vec{A}\right] \times \int_{(\xi+\eta) \epsilon}^{\xi \epsilon} d \vec{A}+4 \vec{\Lambda}(\xi \epsilon-\lambda \epsilon)\left[\int_{(\xi+\eta+\lambda) \epsilon}^{\xi \epsilon} d \vec{A} \cdot \int_{(\xi+\lambda) \epsilon}^{\xi \epsilon} d \vec{A}\right] \\
& +4 \vec{\Lambda}(\xi \epsilon+\eta \epsilon-\lambda \epsilon)\left[\int_{(\xi+\lambda) \epsilon}^{(\xi-\eta) \epsilon} d \vec{A} \cdot \int_{(\xi-\eta) \epsilon}^{(\xi+\eta+\lambda) \epsilon} d \vec{A}\right]+4\left[\vec{\Lambda}(\xi \epsilon-\lambda \epsilon) \cdot \int_{\xi \epsilon}^{(\xi+\eta-\lambda) \epsilon} d \vec{A}\right] \int_{(\xi+\eta+\lambda) \epsilon}^{(\xi+\lambda) \epsilon} d \vec{A} \\
& +4\left[\vec{\Lambda}(\xi \epsilon+\eta \epsilon-\lambda \epsilon) \cdot \int_{(\xi-\lambda) \epsilon}^{(\xi-\eta) \epsilon} d \vec{A}\right] \int_{(\xi+\lambda) \epsilon}^{(\xi+\eta+\lambda) \epsilon} d \vec{A} .
\end{aligned}
$$


Similar to the third order gauge transformation $\delta^{(3)} \vec{A}_{\mu}$ of Eq. (40), we note the appearance of double line integrals in $\delta^{(3)} \psi$, more precisely in $C_{2}$ [Eq. (57)], in $\vec{D}_{2}$ [Eq. (59)], and in $\vec{D}_{3}$ [Eq. (60)]. These double line integrals are defined in Eq. (41) and their properties are discussed in the Appendix.

\section{CONCLUSIONS}

In this paper, we have shown that, at least to third order in the coupling constant, it is possible to construct generalized infinitesimal gauge transformations for Yang-Mills theories incorporating the ideas of point splitting. In contradistinction with the Abelian case [5], we found it necessary to introduce in the gauge transformation a weight function $\rho$ which averages over the splitting. Our analysis shows that the weight function has to satisfy the following conditions.

(1) The function $\rho(\xi)$ has to be a real function of the real variable $\xi$ to preserve the Hermitian character of the gauge fields.

(2) The function $\rho(\xi)$ has to be an even function of $\xi$. This is not only desirable for the sake of simplicity, but also necessary in conjunction with the next property.

(3) The function $\rho(\xi)$ has to satisfy the convolution property (28)

$$
\int_{-\infty}^{+\infty} d \eta \rho(\xi-\eta) \rho(\eta)=\rho(\xi)
$$

to ensure that the generalized gauge transformations could be written in terms of finite line integrals. Also note that the convolution property implies that the weight function cannot be positive definite.

We studied both the case of pure Yang-Mills fields and the case of an SU(2) doublet in interaction with the YangMills fields. In analogy with the Abelian case, we find that the infinitesimal generalized gauge transformations again take the form of a power series in the coupling constant. We worked out in detail these gauge transformations to third order in the coupling constant.

The explicit forms of the generalized gauge transformations for the pure Yang-Mills case are given by Eqs. (5) for zeroth and first orders,

$$
\begin{gathered}
\delta^{(0)} \vec{A}_{\mu}(x)=-\partial_{\mu} \vec{\Lambda}(x), \\
\delta^{(1)} \vec{A}_{\mu}(x)=\int_{-\infty}^{+\infty} d \xi \rho(\xi) \vec{\Lambda}(x+\xi \epsilon) \times \vec{A}_{\mu}(x-\xi \epsilon),
\end{gathered}
$$

by Eq. (30) for order $g^{2}$,

$$
\begin{aligned}
\delta^{(2)} \vec{A}_{\mu}(x)= & \int d \xi \int d \eta \rho(\xi) \rho(\eta)\{\vec{\Lambda}(x+\xi \epsilon+\eta \epsilon) \\
& \times\left[\vec{A}_{\mu}(x-\xi \epsilon) \cdot \int_{x-\xi \epsilon}^{x+(\xi-\eta) \epsilon} d \tau^{\alpha} \vec{A}_{\alpha}(\tau)\right] \\
& -\vec{A}_{\mu}(x+\xi \epsilon+\eta \epsilon) \\
& \times\left[\vec{\Lambda}(x-\xi \epsilon) \cdot \int_{x-\xi \epsilon}^{x+(\xi-\eta) \epsilon} d \tau^{\alpha} \vec{A}_{\alpha}(\tau)\right]+\vec{\Lambda}(x) \\
& \times\left[\int_{x}^{x-\xi \epsilon} d \tau_{1}^{\alpha} \vec{A}_{\alpha}\left(\tau_{1}\right) \cdot \int_{x-\xi \epsilon}^{x+(\xi-\eta) \epsilon} d \tau_{2}^{\beta} \vec{F}_{\mu \beta}\left(\tau_{2}\right)\right] \\
& -\left[\vec{\Lambda}(x-\xi \epsilon) \cdot \int_{x-\xi \epsilon}^{x+(\xi-\eta) \epsilon} d \tau_{2}^{\beta} \vec{F}_{\mu \beta}\left(\tau_{2}\right)\right] \\
& \left.\times \int_{x}^{x+(\xi+\eta) \epsilon} d \tau_{1}^{\alpha} \vec{A}_{\alpha}\left(\tau_{1}\right)\right\}
\end{aligned}
$$

and by Eq. (40) for order $g^{3}$. The corresponding combined gauge parameter is given by Eq. (10) for order $g$,

$$
\vec{L}^{(1)}(x)=\int_{-\infty}^{+\infty} d \xi \rho(\xi) \vec{\Lambda}_{1}(x+\xi \epsilon) \times \vec{\Lambda}_{2}(x-\xi \epsilon),
$$

by Eq. (18) for order $g^{2}$,

$$
\begin{aligned}
\vec{L}^{(2)}(x)= & \int d \xi \int d \eta \rho(\xi) \rho(\eta) \vec{\Lambda}_{1}(x+\xi \epsilon+\eta \epsilon) \\
& \times\left[\vec{\Lambda}_{2}(x-\xi \epsilon) \cdot \int_{x-\xi \epsilon}^{x+(\xi-\eta) \epsilon} d \tau^{\alpha} \vec{A}_{\alpha}(\tau)\right] \\
& -\left(\vec{\Lambda}_{1} \leftrightarrow \vec{\Lambda}_{2}\right)
\end{aligned}
$$

and by Eq. (39) for order $g^{3}$. In the limit $\epsilon \rightarrow 0$ with no point splitting, $\rho$ becomes a $\delta$ function and Eqs. (62) and (64) reduce to the standard Yang-Mills transformations, while the right-hand sides of Eqs. (63), (40), (65), and (39) vanish identically, as expected.

For the case of an $\mathrm{SU}(2)$ doublet coupled to Yang-Mills fields, the generalized gauge transformation, defined by Eq. (44), is given to order $g$ by Eq. (46),

$$
\delta^{(1)} \psi(x)=-\frac{i}{2} \int_{-\infty}^{+\infty} d \xi \rho(\xi) \vec{\tau} \cdot \vec{\Lambda}(x+\xi \epsilon) \psi(x+2 \xi \epsilon),
$$

by Eq. (52) to order $g^{2}$,

$$
\begin{aligned}
\delta^{(2)} \psi(x)= & \int d \xi \int d \eta \rho(\xi) \rho(\eta)\left[-\frac{1}{8}\left\{[\vec{\Lambda}(x+\xi \epsilon)+\vec{\Lambda}(x+2 \xi \epsilon+\eta \epsilon)] \cdot \int_{x+\xi \epsilon}^{x+(2 \xi+\eta) \epsilon} d \vec{A}\right\} \psi(x+2 \xi \epsilon+2 \eta \epsilon)\right. \\
& -\frac{i}{8} \vec{\tau} \cdot\left\{[\vec{\Lambda}(x+2 \xi \epsilon+\eta \epsilon)+\vec{\Lambda}(x+\xi \epsilon+\eta \epsilon)] \times \int_{x+(\xi+\eta) \epsilon}^{x+\xi \epsilon} d \vec{A}\right\} \psi(x+2 \xi \epsilon+2 \eta \epsilon) \\
& -\frac{i}{8} \vec{\tau} \cdot\left\{[\vec{\Lambda}(x+\xi \epsilon+\eta \epsilon)+\vec{\Lambda}(x+\xi \epsilon)] \times \int_{x+(\xi+\eta) \epsilon}^{x+(2 \xi+\eta) \epsilon} d \vec{A}\right\} \psi(x+2 \xi \epsilon+2 \eta \epsilon) \\
& \left.-\frac{i}{4} \vec{\tau} \cdot\left\{[\vec{\Lambda}(x+\xi \epsilon-\eta \epsilon)+\vec{\Lambda}(x+\xi \epsilon)] \times \int_{x+(\xi+\eta) \epsilon}^{x+\xi \epsilon} d \vec{A}\right\} \psi(x+2 \xi \epsilon)\right]
\end{aligned}
$$


and by Eqs. (55)-(60) to order $g^{3}$. Again, Eq. (66) reduces to the standard Yang-Mills transformation in the limit $\epsilon \rightarrow 0$, while Eqs. (67) and (55)-(60) vanish. Taking the limit $\epsilon \rightarrow 0$ is easily seen to coincide with the limit $\rho(\xi) \rightarrow \delta(\xi)$. The weight function $\rho(\xi)$ collapsing to a $\delta$ function eliminates all $\epsilon$ dependence, so the standard Yang-Mills case (without point splitting) is recovered.

In the Abelian case, we were able to show that the gauge transformations can be constructed to arbitrary order. YangMills theories being considerably more complicated, we did not succeed in providing such a proof here, but we see no reason why our analysis could not be extended to higher orders in the coupling constant.

Because the field operators in the generalized gauge transformations are at different space-time points, we can reasonably expect that the corresponding Lagrangian will have regulating properties. We intend to examine this exciting possibility in a forthcoming paper.

\section{ACKNOWLEDGMENTS}

The authors gratefully acknowledge several very useful discussions with their colleagues Professor Harry Lehmann, Professor Per Osland, Professor Walter Troost, and Professor Antoine Van Proeyen. Very special thanks are due to Professor Cecilia Jarlskog for her active interest in our work. The kind hospitality of several institutes, where part of this work was done, is also gratefully acknowledged: C.N. and T.T.W. thank the Theory Division of CERN and the Institute of Physics of the University of Bergen, R.G. thanks the Gordon McKay Laboratory of Harvard University, and R.G. and T.T.W. thank the Department of Physics of the University of Stockholm. The work of C.N. and T.T.W. was supported in part by the U.S. Department of Energy under Grant No. DEFG02-84ER40158.

\section{APPENDIX}

In the third order gauge transformations (39) and (55), we encountered the double line integrals of the form

$$
\begin{aligned}
\iint_{a \epsilon}^{b \epsilon} \vec{A} \times \vec{A}= & \frac{1}{2} \int_{(a+\lambda) \epsilon}^{(b+\lambda) \epsilon} d \tau_{1}^{\alpha} \int_{(a-\lambda) \epsilon}^{(b-\lambda) \epsilon} d \tau_{2}^{\beta}\left[\vec{A}_{\alpha}\left(\tau_{1}\right)\right. \\
& \left.\times \vec{A}_{\beta}\left(\tau_{2}\right)\right]\left[1-\operatorname{sgn}\left(\tau_{1}-\tau_{2}-2 \lambda \epsilon\right)\right] .
\end{aligned}
$$

In this appendix we want to show how they transform under zeroth order gauge transformations. More precisely, we want to show that

$$
\begin{aligned}
\delta^{(0)} \iint_{a \epsilon}^{b \epsilon} \vec{A} \times \vec{A}= & \vec{\Lambda}(b \epsilon-\lambda \epsilon) \times \int_{(a+\lambda) \epsilon}^{(b+\lambda) \epsilon} d \vec{A}+\vec{\Lambda}(a \epsilon+\lambda \epsilon) \\
& \times \int_{(a-\lambda) \epsilon}^{(b-\lambda) \epsilon} d \vec{A}-\int_{a \epsilon}^{b \epsilon} d \tau^{\alpha}[\vec{\Lambda}(\tau+\lambda \epsilon) \\
& \left.\times \vec{A}_{\alpha}(\tau-\lambda \epsilon)+\vec{\Lambda}(\tau-\lambda \epsilon) \times \vec{A}_{\alpha}(\tau+\lambda \epsilon)\right] .
\end{aligned}
$$

To this end, we write

$$
\begin{aligned}
\iint_{a \epsilon}^{b \epsilon} \vec{A} \times \vec{A} & =\int_{(a+\lambda) \epsilon}^{(b+\lambda) \epsilon} d \tau_{1}^{\alpha} \int_{\tau_{1}-2 \lambda \epsilon}^{(b-\lambda) \epsilon} d \tau_{2}^{\beta} \vec{A}_{\alpha}\left(\tau_{1}\right) \times \vec{A}_{\beta}\left(\tau_{2}\right) \\
& =\int_{(a-\lambda) \epsilon}^{(b-\lambda) \epsilon} d \tau_{2}^{\beta} \int_{(a+\lambda) \epsilon}^{\tau_{2}+2 \lambda \epsilon} d \tau_{1}^{\alpha} \vec{A}_{\alpha}\left(\tau_{1}\right) \times \vec{A}_{\beta}\left(\tau_{2}\right)
\end{aligned}
$$

It then follows that

$$
\begin{aligned}
\delta^{(0)} \iint_{a \epsilon}^{b \epsilon} \vec{A} \times \vec{A} \\
=-\int_{(a+\lambda) \epsilon}^{(b+\lambda) \epsilon} d \tau_{1}^{\alpha} \int_{\tau_{1}-2 \lambda \epsilon}^{(b-\lambda) \epsilon} d \tau_{2}^{\beta} \vec{A}_{\alpha}\left(\tau_{1}\right) \times \partial_{\beta} \vec{\Lambda}\left(\tau_{2}\right) \\
\quad-\int_{(a-\lambda) \epsilon}^{(b-\lambda) \epsilon} d \tau_{2}^{\beta} \int_{(a+\lambda) \epsilon}^{\tau_{2}+2 \lambda \epsilon} d \tau_{1}^{\alpha} \partial_{\alpha} \vec{\Lambda}\left(\tau_{1}\right) \times \vec{A}_{\beta}\left(\tau_{2}\right) \\
=-\int_{(a+\lambda) \epsilon}^{(b+\lambda) \epsilon} d \tau_{1}^{\alpha} \vec{A}_{\alpha}\left(\tau_{1}\right) \times[\Lambda(b \epsilon-\lambda \epsilon) \\
\left.-\Lambda\left(\tau_{1}-2 \lambda \epsilon\right)\right]-\int_{(a-\lambda) \epsilon}^{(b-\lambda) \epsilon} d \tau_{2}^{\beta}\left[\Lambda\left(\tau_{2}+2 \lambda \epsilon\right)\right. \\
\quad-\Lambda(a \epsilon+\lambda \epsilon)] \times \vec{A}_{\beta}\left(\tau_{2}\right),
\end{aligned}
$$

which is the same as Eq. (A2).

It should be noted that the double integrals of the type (A1) always appear in the gauge transformations integrated over $\lambda$ with the weight function $\rho(\lambda)$. The evenness of $\rho(\lambda)$ then allows us to obtain the simpler result that

$$
\begin{gathered}
\delta^{(0)} \int d \lambda \rho(\lambda) \iint_{a \epsilon}^{b \epsilon} \vec{A} \times \vec{A} \\
=\int d \lambda \rho(\lambda)\{[\vec{\Lambda}(b \epsilon-\lambda \epsilon)+\vec{\Lambda}(a \epsilon-\lambda \epsilon)] \\
\left.\quad \times \int_{(a+\lambda) \epsilon}^{(b+\lambda) \epsilon} d \vec{A}-2 \int_{a \epsilon}^{b \epsilon} d \tau^{\alpha} \vec{\Lambda}(\tau+\lambda \epsilon) \times \vec{A}_{\alpha}(\tau-\lambda \epsilon)\right\} .
\end{gathered}
$$

The last integral in Eq. (A5) is precisely

$$
-2 \int_{a \epsilon}^{b \epsilon} \delta^{(1)} \vec{A}
$$

as can be seen by comparing with Eq. (35). It thus follows from this exercise that the double line integrals of the type (A1) are essential for the cancellation of the terms involving integrals over $\delta^{(1)} \vec{A}$. 
[1] C.N. Yang and R.L. Mills, Phys. Rev. 96, 191 (1954).

[2] P.A.M. Dirac, Proc. Cambridge Philos. Soc. 30, 150 (1934).

[3] R. Peierls, Proc. R. Soc. London A146, 420 (1934); J. Schwinger, Phys. Rev. 82, 664 (1951); K. Johnson, in Lectures on Particles and Field Theory, Lectures at Brandeis University 1964, edited by S. Deser and K.W. Ford (PrenticeHall, Englewood Cliffs, NJ, 1965), Vol. 2, p. 1.
[4] P. Osland and T.T. Wu, Z. Phys. C 55, 569 (1992); 55, 585 (1992); 55, 593 (1992); Phys. Lett. B 291, 315 (1992); C. Newton, P. Osland, and T.T. Wu, Z. Phys. C 61, 441 (1994); C. Newton and T.T. Wu, ibid. 62, 253 (1994).

[5] R. Gastmans, C. Newton, and T.T. Wu, CERN Report No. CERN-TH.7008/93 (unpublished); Phys. Lett. B 341, 84 (1994). 